\title{
Shell tectonics: A mechanical model for strike-slip displacement on Europa
}

\author{
Alyssa Rose Rhoden ${ }^{\mathrm{a}, *, 1}$, Gilead Wurman ${ }^{\mathrm{b}}$, Eric M. Huff ${ }^{\mathrm{c}}$, Michael Manga ${ }^{\mathrm{a}}$, Terry A. Hurford ${ }^{\mathrm{d}}$ \\ ${ }^{a}$ University of California at Berkeley, Department of Earth E Planetary Science, Berkeley, CA 94720, United States \\ ${ }^{\mathrm{b}}$ Seismic Warning Systems, Inc., Scotts Valley, CA 95066, United States \\ ${ }^{\mathrm{c}}$ University of California at Berkeley, Department of Astronomy, Berkeley, CA 94720, United States \\ ${ }^{\mathrm{d}}$ NASA Goddard Space Flight Center, Code 693, Greenbelt, MD 20771, United States
}

\section{A R T I C L E I N F O}

\section{Article history:}

Received 11 April 2011

Revised 17 November 2011

Accepted 19 December 2011

Available online 29 December 2011

\section{Keywords:}

Europa

Tectonics

Rotational dynamics

\begin{abstract}
A B S T R A C T
We introduce a new mechanical model for producing tidally-driven strike-slip displacement along preexisting faults on Europa, which we call shell tectonics. This model differs from previous models of strike-slip on icy satellites by incorporating a Coulomb failure criterion, approximating a viscoelastic rheology, determining the slip direction based on the gradient of the tidal shear stress rather than its sign, and quantitatively determining the net offset over many orbits. This model allows us to predict the direction of net displacement along faults and determine relative accumulation rate of displacement. To test the shell tectonics model, we generate global predictions of slip direction and compare them with the observed global pattern of strike-slip displacement on Europa in which left-lateral faults dominate far north of the equator, right-lateral faults dominate in the far south, and near-equatorial regions display a mixture of both types of faults. The shell tectonics model reproduces this global pattern. Incorporating a small obliquity into calculations of tidal stresses, which are used as inputs to the shell tectonics model, can also explain regional differences in strike-slip fault populations. We also discuss implications for fault azimuths, fault depth, and Europa's tectonic history.
\end{abstract}

() 2011 Elsevier Inc. All rights reserved.

\section{Introduction}

Strike-slip offsets are common along Europan lineaments of varying length and type (Schenk and McKinnon, 1989; Tufts et al., 1999; Hoppa et al., 2000; Figueredo and Greeley, 2000; Sarid et al., 2002; Kattenhorn, 2002; Riley et al., 2006; Kattenhorn and Hurford, 2009). A comprehensive survey of strike-slip faults in the Galileo regional mapping data set identified almost 200 strike-slip faults (Sarid et al., 2002). In Fig. 1, we show the azimuths and locations of the left lateral (red/dark gray) and right lateral (blue/light gray) faults that were identified. The survey revealed that, on a large-scale, the sense of motion along faults is not random. The distribution of faults is generally left lateral (LL) in the far north and right lateral (RL) in the far south, with a mixture of both fault types in between. The ratio of LL to RL faults in the mixed region decreases with latitude and trends differently between the leading and trailing hemispheres. Fault statistics from the survey (Sarid et al., 2002) are shown in more detail in Table 1.

As Europa moves through its eccentric orbit, the magnitude and direction of its Jupiter-raised tidal bulges change. The result is daily-varying deformation and stress that can drive tectonics. Tidal stress due to orbital eccentricity has been linked to the formation

\footnotetext{
* Corresponding author.

E-mail address: alyssa@eps.berkeley.edu (A.R. Rhoden).

${ }^{1}$ Previously published under the surname Sarid.
}

of several types of tectonic features on Europa including strike-slip faults (Tufts et al., 1999; Hoppa et al., 2000; Kattenhorn, 2002; Sarid et al., 2002; Rhoden et al, 2011). The motion and eruption timing along the tiger stripe fractures on Enceladus (Hurford et al., 2007; Nimmo et al., 2007; Smith-Konter and Pappalardo, 2008) have also been attributed to tides caused by Enceladus' eccentricity and close proximity to Saturn.

Additional orbital and rotational characteristics of these satellites can also influence the pattern of stress change throughout each orbit. Gravitational interactions between Jupiter's large satellites cause Europa's obliquity to be at least $0.1^{\circ}$, and possibly larger if the satellite has a subsurface ocean (Bills et al., 2009). Evidence of $\sim 1^{\circ}$ of obliquity has been found within Europa's geologic record, preserved in the shapes of arcuate features called cycloids (Hurford et al., 2009; Rhoden et al., 2010). Rhoden et al. (2010) found that good fits to cycloids were achieved only when longitude translation was assumed, supporting the idea that Europa's shell rotates non-synchronously (Rhoden et al., 2010). However, cycloid fits did not indicate that stress from non-synchronous rotation (NSR) influences their formation. Non-synchronous rotation of Europa's ice shell has been proposed on theoretical grounds (Greenberg and Weidenschilling, 1984; Ojakangas and Stevenson, 1989) and is supported by geologic evidence (see review in Bills et al. (2009)). However, if the rotation is slow, the accumulation of stress from non-synchronous rotation may be limited (Greenberg et al., 1998; Goldreich and Mitchell, 2010). 


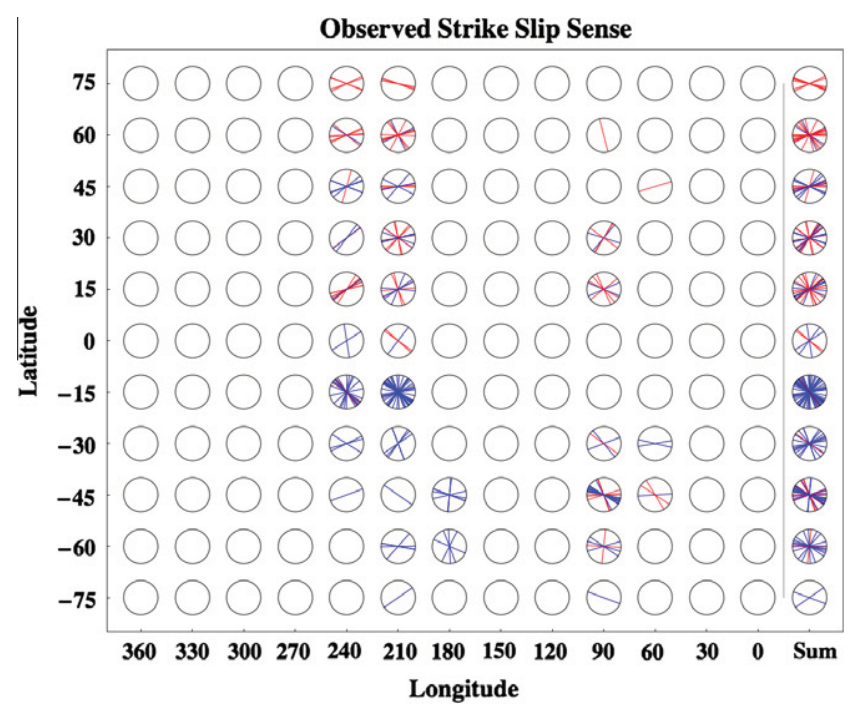

Fig. 1. Observed faults from the survey by Sarid et al. (2002) shown at their measured azimuths. Left-lateral faults are blue (dark gray), and right-lateral faults are red (light gray). The faults are binned in latitude and longitude. At longitude $90^{\circ} \mathrm{W}$ and latitudes $0^{\circ}$ and $15^{\circ} \mathrm{S}$, pervasive chaos formation inhibited fault identification. Other empty circles represent regions that were not mapped due to limited high-resolution imagery. (For interpretation of the references to color in this figure legend, the reader is referred to the web version of this article.)

Table 1

Strike-slip statistics from Sarid et al. (2002).

\begin{tabular}{|c|c|c|c|c|c|c|}
\hline & \multicolumn{3}{|c|}{ Trailing hemisphere } & \multicolumn{3}{|c|}{ Leading hemisphere } \\
\hline & LL & $\mathrm{RL}$ & $\% \mathrm{LL}$ & LL & $\mathrm{RL}$ & \% LL \\
\hline $60 \mathrm{~N}+$ & 18 & 0 & 100 & - & - & - \\
\hline $40-60 \mathrm{~N}$ & 8 & 6 & 57 & 2 & 0 & 100 \\
\hline $20-40 \mathrm{~N}$ & 17 & 13 & 57 & 4 & 1 & 80 \\
\hline $0-20 \mathrm{~N}$ & 1 & 4 & 20 & 3 & 2 & 60 \\
\hline $20 S^{a}-0$ & 7 & 47 & 13 & - & - & - \\
\hline $40-20 S$ & 0 & 8 & 0 & 3 & 5 & 38 \\
\hline $60-40 S$ & 0 & 11 & 0 & 8 & 16 & 33 \\
\hline $60 S_{-}$ & 0 & 7 & 0 & 0 & 1 & 0 \\
\hline
\end{tabular}

a The southernmost LL fault in the trailing hemisphere is located at $21.4^{\circ} \mathrm{S}$; we included it in the $0-20^{\circ} \mathrm{S}$ bin.

The tidal stress due to orbital eccentricity and obliquity can be decomposed into normal and shear components along a fault of given azimuth and location (Section 3.1). Depending on the fault response model adopted, the changes in stress throughout an orbit can be analyzed to determine the likely net offset direction along the fault. We present a new mechanical model for the formation of these tidally-driven strike-slip faults (Sections 2.2 and 3.2). Our "shell tectonics" model combines tidal stress and an elastic rebound seismic model for slip and stress release on faults, in which accumulated shear stress on the fault is released when the fault slips. Our model successfully reproduces the observed global pattern of strike-slip faults on Europa. When obliquity is also incorporated, we can generate variations in the global pattern that are consistent with the observed differences in the leading and trailing hemisphere strike-slip fault populations (Sections 4 and 5.1). In contrast, we find that a model neglecting the release of stress due to slip is less effective at matching the global pattern of strike-slip (Sections 4 and 5.1). We also discuss implications of these results on relative offset accumulation rates along faults and the depth to which strike-slip motion may occur (Sections 5.2 and 5.3). In addition to advancing our understanding of tidally-driven tectonics on Europa, the shell tectonics model may also be relevant for Enceladus. Strike-slip motion and subsequent heat generation along the Tiger Stripe fractures has been linked to tides (Nimmo et al., 2007; Smith-Konter and Pappalardo, 2008), and tidally-driven opening and closing of the fractures has been proposed as a mechanism for controlling plume eruptions (Hurford et al., 2007).

\section{Models of tidally-controlled strike-slip}

\subsection{Previous models}

As Europa moves through its eccentric orbit, a pre-existing fault will experience diurnally-varying normal and shear stresses that may drive tectonics. Hoppa et al. (1999, 2000) introduced the tidal walking model, which assumes that faults slip freely while in tension, but that slip is prohibited during compression. Once failure initiates, slip on the fault accumulates until the magnitude of the tidal shear stress reaches a maximum. Then, because the ice shell is assumed to behave elastically over short (i.e. diurnal) timescales, slip on the fault is reduced back toward zero as the shear stress decreases. Therefore, if the shear stress crosses zero during the tension phase, it is assumed that any accumulated slip up to that point has been recovered, and there is no net offset on the fault. When the normal stress becomes compressive, the fault is clamped; an offset on the fault reflects the amount of accumulated slip since the last time the shear stress crossed zero. In order to maintain this offset over subsequent orbits, elastic stresses in the shell must relax while the fault is clamped so that, when the normal stress again becomes tensile, the fault will not slip back to its initial configuration.

Hoppa et al. (1999, 2000) used the tidal walking model to create global predictions of slip direction, which generally match the observed global pattern on Europa. Rhoden et al. (2011) made a correction to the predictions, but this did not affect the resulting global pattern of only left lateral (LL) faults poleward of $35^{\circ} \mathrm{N}$, only right lateral (RL) faults poleward of $35^{\circ} \mathrm{S}$, and between these regions, either right or left lateral faults with the slip-direction depending on the longitude and the azimuth of the crack. Initially, observed differences in the strike-slip statistics of the leading and trailing hemispheres were attributed to non-synchronous rotation and true polar wander (Sarid et al., 2002). However, Rhoden et al. (2011) investigated the effects of obliquity on the predictions and concluded that an obliquity of $\sim 1^{\circ}$ could lead to offsets of the regions of mixed right and left lateral faults that are more consistent with the observations than polar wander.

Its ability to reproduce the observed global pattern of strike-slip displacement is a strong indication that the tidal walking model captures a fundamental attribute of the fault slip process on Europa. However, the mechanics of the model are simplified. Neither stress release due to slip nor stress relaxation with time are explicitly included in the tidal walking model. In fact, predictions of slip direction are based solely on the sign of the shear stress when the normal stress becomes compressive (see Rhoden et al., 2011). Furthermore, failure is only allowed when the fault experiences tensile normal stress so the model is only applicable in the upper tens of meters of Europa's surface. Deeper within the shell, the compressive overburden stress would exceed the tidal stress and clamp the fault throughout the entire orbit.

A model for strike-slip formation due to tidal stress has also been proposed for Enceladus (Smith-Konter and Pappalardo, 2008) and should be equally applicable to Europa. In this model, which we will refer to as the SKP model, the fault can slip whenever the tidal shear stress exceeds the Coulomb failure criterion. Slip on the fault accumulates via a large event when the shear stress exceeds the Coulomb failure threshold and subsequent small, creeping events that continue as long as the tidal shear stress remains above the failure threshold. Summing the accumulated slip over one orbit yields a prediction of slip direction. 
Comparison between model predictions and the observed tiger stripe fractures on Enceladus is inconclusive (Smith-Konter and Pappalardo, 2008), and the model has not yet been tested against the global pattern of strike-slip faults on Europa.

A major difference between the tidal walking model and the SKP model is in the determination of slip direction throughout an orbit. In the tidal walking model, it is assumed that the shell, being elastic over short timescales, will act to restore the fault to its initial configuration. Hence, slip depends on the change in shear stress with time. In the SKP model, fault slip is determined by the value of the tidal shear stress regardless of whether it is increasing or decreasing. One way to visualize the difference is to think of the SKP model as describing the behavior along a boundary between two unconnected plates. Whereas, in the tidal walking model, the plates are connected in the far field by a spring. It is unclear a priori, which model best represents the behavior of faults on Europa and Enceladus.

To further investigate the mechanism of strike-slip fault formation on Europa, we take a dual approach. First, we develop a model based on the assumption of elastic rebound over short timescales, in the spirit of the tidal walking model, but that also includes a more quantitative and physical treatment of stress accumulation and slip on a fault. We also create a simplified version of the SKP model, adopting the rules for stress accumulation and slip outlined by Smith-Konter and Pappalardo (2008) and developed with their guidance (Smith-Konter, personal communication, 2010). We then test the underlying fault slip assumptions of each model by creating predictions of fault slip direction and comparing the predictions against the observations in the strike-slip survey by Sarid et al. (2002).

\subsection{Shell tectonics}

In the shell tectonics model, slip occurs when the accumulated shear stress on the fault exceeds the Coulomb failure threshold. When a fault is in tension, we set the failure criterion to zero (no cohesion). This results in continuous slip while the fault is in tension and large discrete events in compression. As with the SKP model for Enceladus (Smith-Konter and Pappalardo, 2008), allowing slip while the fault is under compression allows the shell tectonics model to operate deeper in the ice shell than the tidal walking model.

When the failure threshold is exceeded, the amount of resulting slip is assumed to be proportional to the accumulated shear stress on the fault at that time. We explicitly include the release of accumulated shear stress due to slip by resetting the shear stress on the fault to zero after every slip event. Hence, the tidal shear stress has to increase in magnitude for the fault to continue slipping in the same direction. If the shear stress instead decreases, the fault will begin to slip back toward its initial configuration. Thus, release of accumulated stress accounts for the elastic response of the shell. This is in contrast to the SKP model in which the fault continues to creep after the failure threshold is reached, and the direction of creep is always in the same direction as the initial slip event. To account for the long-term viscous behavior of Europa's ice shell, we also allow a small amount stress of relaxation when on the fault, which we find to be critical to producing net offsets. To make a prediction of slip direction, we determine the long-term trend of motion on the fault by calculating the difference in the net offset over many orbits. Additional details of these calculations are given in Section 3.2.

Use of accumulated shear stress rather than tidal shear stress when determining failure is a keystone of the shell tectonics model. Fig. 2 is a schematic showing the tidal deformation of an unslipped region and a fault's response to that deformation at certain points in the orbit. The direction of the most compressive principal stress is also shown. Throughout the schematic, there is a regional left lateral (LL) tidal shear stress, which is increasing in magnitude at $A$ and $B$ but decreasing thereafter. We also assume that the regional tidal normal stress has just entered a period of compression. Previously, the fault was free to slip in response to the left lateral tidal deformation because it was in tension.

At A, the fault has a LL offset and zero accumulated shear stress. At $B$, the tidal deformation in the region has become increasingly left lateral. Based solely on the regional deformation, the fault would accumulate another LL offset, even larger than at A. However, if we only consider the accumulated stress on the fault, we get a much different answer. Because, the fault has already responded to the deformation at $\mathrm{A}$, it accumulates a small amount of additional shear stress due to the incremental increase in deformation, but it is not enough to reach the failure threshold. At $C$, the tidal shear stress has decreased and is now identical to A. Thus, the accumulated shear stress on the fault returns to zero. At D, the regional deformation has decreased further. If the fault had never slipped, we would expect the deformation to induce left-lateral slip on the fault as long as the tidal stress exceeded the failure criterion. However, the fault has already slipped to accommodate the deformation at $C$, which is now more LL slip than would be generated at D. Hence, at D, the fault actually accumulates right lateral shear stress. At E, the deformation has decreased so much that
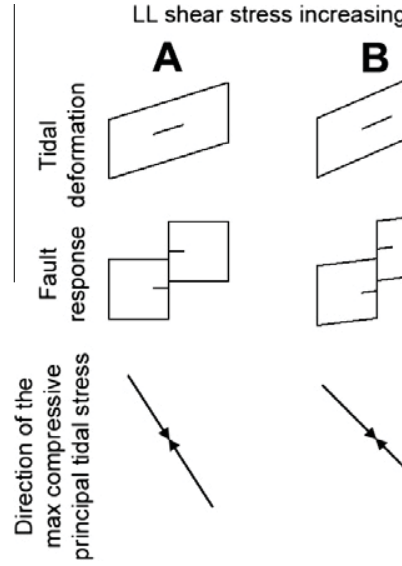
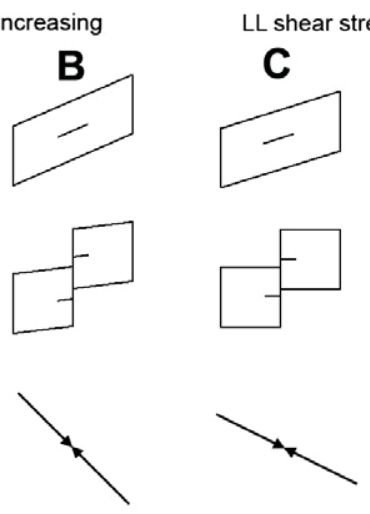

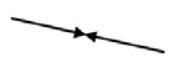

\section{D}
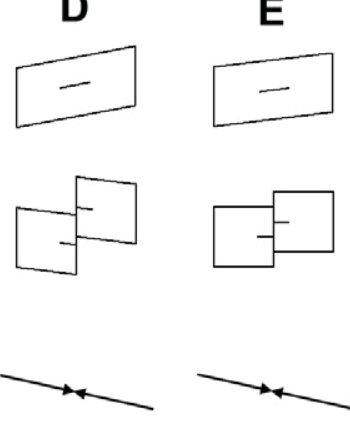

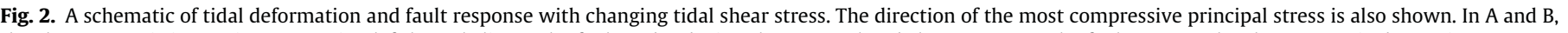

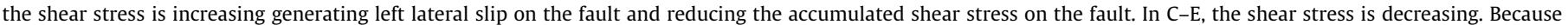

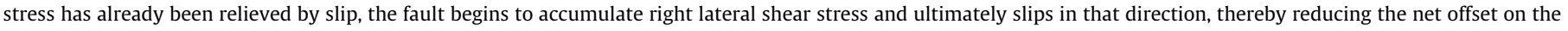
fault. 
the accumulated shear stress on the fault generates right lateral slip even though the regional tidal shear stress is still left lateral. The shell tectonics model would thus predict left lateral slip at A and right lateral slip at E. In the SKP model, the fault would undergo a large LL slip at A, continue creeping in a LL sense until C, and not slip again in this time period because the tidal shear stress is lower than the failure threshold. In the tidal walking model, no slip would have occurred during this entire time period because the fault was in compression.

\section{Generating global predictions}

\subsection{Calculating tidal stress}

Our formation model is based on the hypothesis that tidal deformation is the driver of strike-slip displacement on Europa. The daily changes in tidal stress thus control the ability of preexisting faults to slip and the direction of net shear displacement along the faults. We neglect stress from non-synchronous rotation or overburden pressure (see also, Section 5.3). We assume that Europa has long-since adopted its primary tidal shape, which is based on the average distance and angle between Europa and Jupiter. It is deviations away from that shape that generate stress. We use the equations for stress in a thin, elastic shell (e.g., Melosh, 1977, 1980):

$\sigma_{\delta}=C\left(5+3 \cos 2 \delta_{P}\right)$

$\sigma_{\alpha}=-C\left(1-9 \cos 2 \delta_{P}\right)$

where $C=3 h_{2} M \mu(1+v) / 8 \pi \rho a^{3}(5+v)$ and $h_{2}$ is Europa's tidal Love number, $M$ is Jupiter's mass, $\mu$ is Europa's shear modulus, $v$ is the Poisson's ratio of Europa, $\rho$ is Europa's average bulk density, a is the average distance between Jupiter and Europa, and $\delta_{P}$ is the angular distance from a point on Europa's surface to its primary tidal bulge. The $\sigma_{\delta}$ stress is directed radially from the tidal bulges and the $\sigma_{\alpha}$ stress is perpendicular to $\sigma_{\delta}$. Because all the stress calculations contain a factor of $C$, its value does not influence our results.

The tidal stress equations can be modified to account for Europa's eccentricity and obliquity (Hurford et al., 2009; Rhoden et al., 2010,2011 ). Eccentricity causes the tidal bulges to librate in longitude while obliquity predominantly causes a latitudinal libration. Spherical trigonometry is used to calculate the time-varying location of the tidal bulge, which now depends on the spin pole direction and the true anomaly. Because the locations of the tidal bulges are changing, the angular distance to the bulge, $\delta$, also changes. The equations then become:

$\sigma_{\delta}=C(1-e \cos n)^{-3}(5+3 \cos 2 \delta)$

$\sigma_{\alpha}=-C(1-e \cos n)^{-3}(1-9 \cos 2 \delta)$

and

Bulge colatitude $=\pi / 2-\varepsilon \sin (n+\varphi)$

Bulge longitude $=-2 e \sin n$

where $e$ is Europa's eccentricity, $\varepsilon$ is Europa's obliquity, $\varphi$ is the spin pole direction (SPD), and $n$ is the true anomaly. When Europa is at pericenter, if the spin pole is pointing toward Jupiter, the SPD is defined as $90^{\circ}$; SPD increases clockwise. There is degeneracy between spin pole direction and longitude such that the stress field is identical when both are modulated by $180^{\circ}$.

Stress from the diurnal tide is calculated by subtracting the primary tidal stress (Eqs. (1a) and (1b)) from the stress due to both eccentricity and obliquity (Eqs. (2a) and (2b)) once the stresses have been rotated to a common coordinate system. The resulting diurnal stresses in the new coordinate system are $\sigma_{\delta *}$ in the direction of the north pole, and the perpendicular stress, $\sigma_{\alpha *}$ There may now also be a shear stress, $\sigma_{\delta \alpha *}$ These diurnal tidal stresses can then be decomposed into normal and shear components relative to a fault's orientation, where $\zeta$ is the azimuth of the crack measured clockwise from north.

$\sigma=0.5\left(\sigma_{\delta *}+\sigma_{\alpha * *}\right)+0.5\left(\sigma_{\delta *}-\sigma_{\alpha *}\right) \cos (2 \zeta)+\sigma_{\delta \alpha *} \sin (2 \zeta)$

$\tau=-0.5\left(\sigma_{\delta *}-\sigma_{\alpha *}\right) \sin (2 \zeta)+\sigma_{\delta \alpha *} \cos (2 \zeta)$

To determine the slip direction along a fault, we first specify the latitude and longitude of the fault, the fault azimuth, and the prescribed amount of obliquity and spin pole direction. Using these parameters, we calculate the stress throughout an orbit.

The equations for tidal stress shown here assume an elastic rather than viscoelastic shell. Wahr et al. (2009) present equations for tidal stress in a viscoelastic shell. However, the Wahr et al. (2009) model does not include obliquity, an important parameter in our work. In addition, the predictions made with the tidal walking model relied on the formulation we present here (Hoppa et al., 1999, 2000; Rhoden et al., 2011), so it is useful for consistency to test shell tectonics against the same stresses. Smith-Konter and Pappalardo (2008) did use the Wahr et al. (2009) model, so it is important to consider how the two approaches differ in their calculations of tidal stress.

A major result of Wahr et al. (2009) is that stresses caused by non-synchronous rotation in a viscoelastic shell are phase-shifted from those in an elastic shell. For diurnal stresses, the amplitudes can differ by $0.1-3 \%$ between the elastic and viscoelastic cases, depending on the viscosity assumed for the ice shell. Because we neglect the effects of NSR stress, the differences between the diurnal stresses we calculate here and those used by Smith-Konter and Pappalardo (2008) should be small - indeed, no larger than 3\%. Our main goal is to test whether observations are best explained with the assumption that - quite generally - slip on a fault is reduced when the shear stress decreases (shell tectonics) or that slip continues to increase even if the shear stress decreases (SKP). The exact amplitude of the tidal stress will not significantly affect this determination.

\subsection{Applying shell tectonics}

We use the shell tectonics model to fit the global strike-slip pattern on Europa by generating predictions on a global scale. We determine net offsets for faults with azimuths from $0^{\circ}$ to $180^{\circ}$ in $1^{\circ}$ increments, at longitudes from $0^{\circ}$ to $360^{\circ}$ every $30^{\circ}$, and from latitude $75^{\circ}$ to $-75^{\circ}$ in increments of $15^{\circ}$. We apply shell tectonics to tidal stress fields with and without $1^{\circ}$ of obliquity and test two end-member values of the friction coefficient: 0.6 and 0.2 . The higher friction value is appropriate for rock and may be applicable to very cold ice (Beeman et al., 1988); the lower value was used by Smith-Konter and Pappalardo (2008). We use it here for consistency.

To calculate the accumulated stress on the fault, we solve the following differential equation in which $\boldsymbol{\sigma}_{\text {tidal }}$ is the stress tensor and stress relaxes on an e-folding timescale of $1 / \eta$ :

$d \boldsymbol{\sigma}_{\mathrm{acc}} / d t=\left(d \boldsymbol{\sigma}_{\mathrm{tidal}} / d t\right)-\eta \boldsymbol{\sigma}_{\mathrm{acc}}$

The relaxation rate is a depth-averaged value that accounts for the decrease in ice viscosity with depth. Our solution is restricted to values of $\eta$ for which $\eta t_{\text {orbit }} \ll 1$. Without relaxation, we find that no net slip accumulates over time.

There is no release of accumulated normal stress in this model. Rather, we assume that the accumulated normal stress on the fault reflects the long-term behavior of daily-varying stresses. We analytically solve Eq. (4) to determine the initial values of the accumulated normal stress components such that the transient part of the solution is minimized for each component. We also advance in the 
orbit to a time when the fault is in tension so the initial value of the accumulated shear stress can be set to zero. At each subsequent time step, we first add $\Delta \boldsymbol{\sigma}_{\text {tidal }}$ and calculate the normal and shear components of the accumulated stress (using Eqs. (3a) and (3b)). If the accumulated normal stress on the fault is positive (in tension), it will slip for any non-zero value of accumulated shear stress. In compression $\left(\sigma_{\text {acc }}<0\right)$, the accumulated shear stress must exceed the Coulomb failure threshold.

$\tau_{\mathrm{acc}}=-f \sigma_{\text {acc }}$

where $f$ is the friction coefficient. If slip occurs, the cumulative slip is increased by the amount of accumulated stress on the fault and the accumulated shear stress is set to zero. Finally, we reduce the deviatoric component of any remaining accumulated stress, $\boldsymbol{\sigma}_{\text {acc }}$, according to Eq. (4) to account for the long-term viscoelastic behavior of the shell. We compute the accumulated stress and cumulative slip at 8500 time steps per orbit and run each simulation over 1000 orbits to ensure that the solution has converged.

To predict the slip direction on the fault over time, we calculate the difference in slip at the same point in two successive orbits to determine the net offset. We then average the net offset over 1000 orbits to determine the long-term behavior on the fault. To reduce numerical noise, we repeat the simulation 20 times with a random number (between 0 and 1 ) added to the longitude, and average the net offset values over the 20 simulations. If the average net offset is positive, we predict left-lateral displacement on the fault; a negative average net offset yields a right-lateral prediction. If the net offset averages to zero, we predict no net slip on the fault.

\subsection{Applying an SKP-type model}

Because the observed global pattern is such a useful metric for testing tidal-tectonic models, we also generate predictions using a model based on Smith-Konter and Pappalardo (2008). In that model, there is a large slip event when the fault first reaches the failure criterion. Then, small slips occur as long as the tidal stress on the fault exceeds the Coulomb failure threshold. The amount of slip from the large events is proportional to the tidal shear stress on the fault at the time of failure. For the creeping events, Smith-Konter and Pappalardo (2008) assume a constant, time-averaged, strain rate to which the amount of accumulated slip is proportional. Unlike tidal walking or shell tectonics, the direction of slip in the SKP model is always the same as the tidal shear stress direction. The actual model presented in Smith-Konter and Pappalardo (2008) is more sophisticated than what we are using here. However, our interest is in testing the determination of when and how fault slip will occur in the SKP model versus the shell tectonics model. To accomplish this, we have adopted a set of rules for stress accumulation and fault slip that capture these fundamental assumptions.

To compute a prediction of direction using our SKP-type model, we keep a running sum of LL (positive) and RL (negative) slip throughout an orbit. When the tidal stress on a fault first reaches the failure criterion for one slip direction, there is a large slip event in which all of the tidal stress on the fault is relieved. Hence, the amount of slip is proportional to the amount of shear stress. Subsequently, we calculate the incremental change in stress at each time step within the slip window; the rate of change of the stress is proportional to the slip rate and can thus be used to determine additional slip. Eventually, the shear stress becomes lower than the failure threshold and no slip occurs on the fault. Later, the fault may slip in the opposite direction reducing the total offset on the fault. After one orbit, positive cumulative slip would mean a LL prediction for the fault, negative would be RL, and zero net slip implies no offset on the fault. In practice, we find that the failure stress in each slip direction is similar so the prediction is most dependent on the length of the slip window in each direction. Only in cases

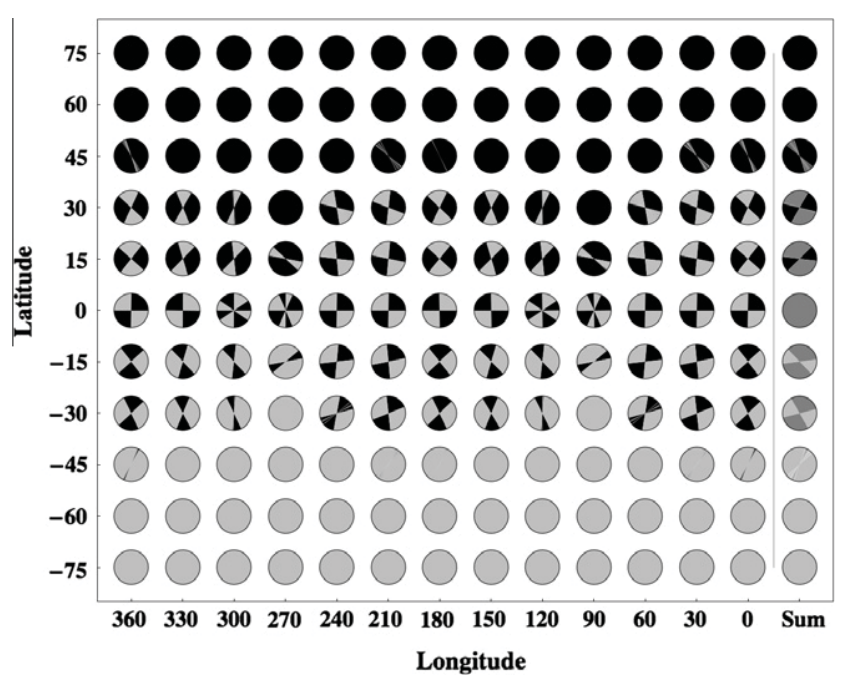

Fig. 3. Shell tectonics predictions of slip direction with zero obliquity. Within each circle, black regions indicate crack azimuths along which we predict left lateral displacement; light gray represents right lateral fault azimuths. We predict no net slip at the boundaries between left and right regions. The last column shows the predictions summed over all longitudes, in which dark gray represents azimuths that could have right or left lateral displacement depending on their longitude at the time the displacement occurred. The pattern of slip direction generated using the shell tectonics model fits with the global observed pattern, in which left-lateral faults dominate in the far north, right lateral faults dominate in the far south, and there is a mixture of right and left lateral faults in between.

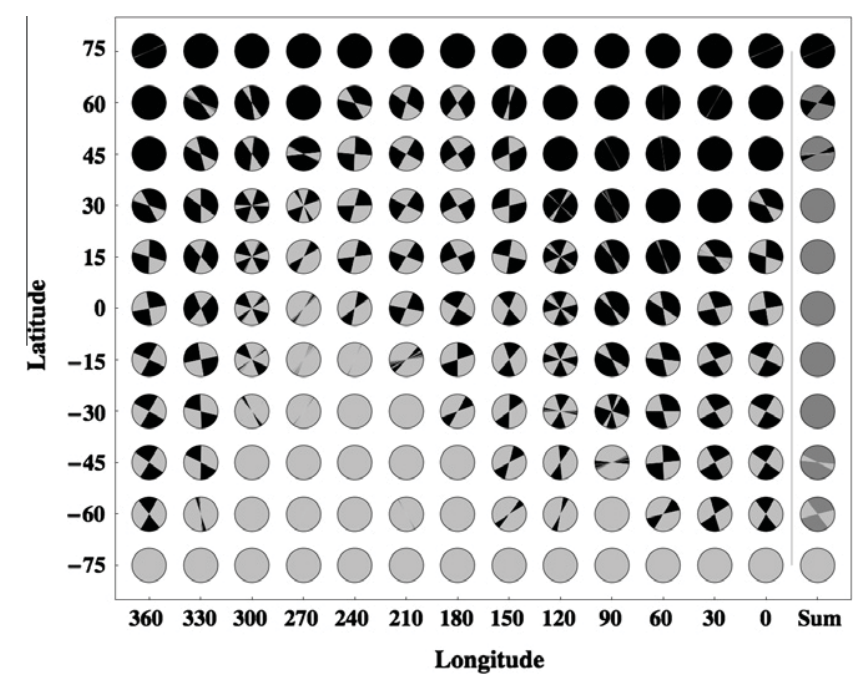

Fig. 4. Shell tectonics predictions of slip direction with $1.0^{\circ}$ obliquity at a spin pole direction of $220^{\circ}$. Black represents fault azimuths that will exhibit left lateral displacement, and light gray represents right lateral faults. The last column shows the predictions at all longitudes combined, in which dark gray indicates that either type of fault can form over the span of longitudes at which we calculated predictions. Obliquity breaks the longitudinal symmetry in the pattern of predicted slip direction, which is consistent with the observed differences in the observed fault populations of the leading and trailing hemispheres.

in which the fault spends equal time in LL and RL slip does the amount of initial slip play a role. This result is consistent with the results of Smith-Konter and Pappalardo (2008).

\section{Results}

The shell tectonics global predictions accounting for only eccentricity are shown in Fig. 3. The predictions using tidal stresses that include a $1^{\circ}$ obliquity and spin pole direction of $220^{\circ}$ are shown in 
Fig. 4. Both sets of predictions use the lower friction value of 0.2 ; predictions using the higher friction value are shown in the Electronic supplement. In these plots, black represents fault azimuths that undergo left lateral slip, and light gray represents right lateral slip. The net slip goes to zero at transitions between left and right lateral regions. In the last column of each plot, we show a combination of the predictions made at each latitude over all longitudes. Here, dark gray indicates that both right and left lateral faults form within the span of longitudes tested for a given crack azimuth. To generate the predictions shown in Figs. 3 and 4, we use a relaxation rate of $1 \times 10^{-10} \mathrm{~s}^{-1}$, which is comparable to the Maxwell time of ice at $\sim 130 \mathrm{~K}$. The relaxation rate will determine the magnitude of the accumulated offset per orbit. However, our predictions of slip direction and relative offset accumulation are insensitive to the relaxation rate over several orders of magnitude (see online Supplement for an example).

Without obliquity (Fig. 3), the shell tectonics model predicts regions with only left lateral faults at latitudes $60^{\circ}$ and $75^{\circ}$, and right lateral faults at $-60^{\circ}$ and -75 . Latitudes $45^{\circ}$ and $-45^{\circ}$ are nearly exclusive, but there are a few longitudes with very narrow azimuth ranges that predict the opposite slip direction. At longitude $90^{\circ} \mathrm{W}$ and $270^{\circ} \mathrm{W}$, on the other hand, the regions of exclusivity extend toward the equator to include $\pm 30^{\circ}$. At all other latitudes, there is a mixture of RL and LL faults predicted at every longitude. Using the higher friction value $(0.6)$ produces very similar global predictions for this case, as shown in the Electronic supplement.

With obliquity, a distinct hemispheric pattern emerges, as shown in Fig. 4 (for $\varphi=220^{\circ}$ ). In the trailing hemisphere, around longitude $210^{\circ} \mathrm{W}$, exclusively LL regions are predicted farther north than without obliquity while exclusively RL regions are predicted closer to the equator. In the leading hemisphere (lon $90^{\circ} \mathrm{W}$ ), the opposite occurs: LL-only regions begin farther south, and mixed regions extend into lower latitudes than without obliquity. With the higher friction value (see SOM), there are similar trends, but fewer locations are predicted to have exclusively LL or RL faults for all azimuths. The spin pole direction determines the longitudes at which the asymmetries in predictions would occur. Qualitatively, the pattern of offsets produced by obliquity appears consistent with the hemispheric differences in the observed strike-slip populations.

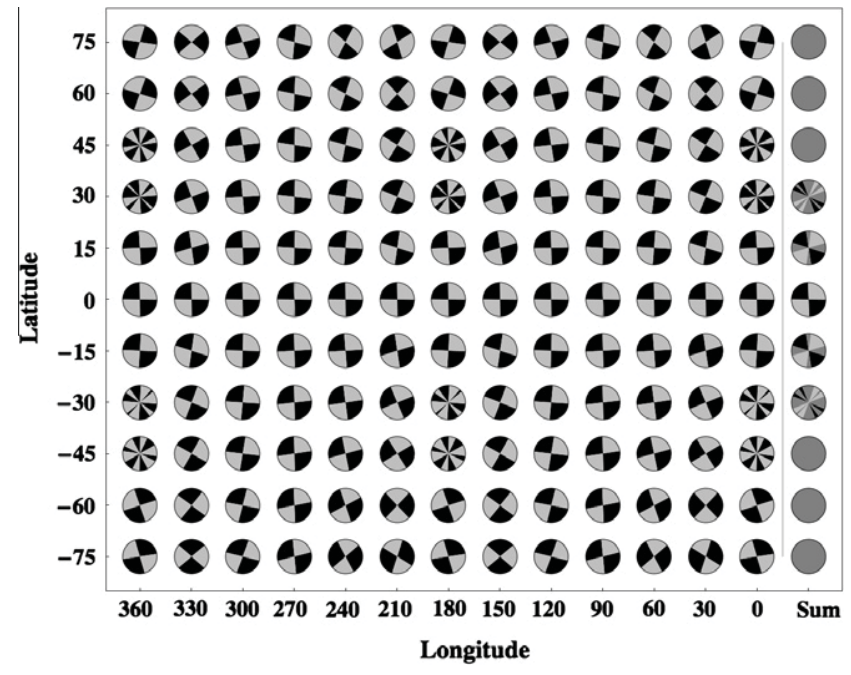

Fig. 5. Model predictions of slip direction with zero obliquity using the SKP-type model. Black represents fault azimuths that will exhibit left lateral displacement, and light gray represents right lateral faults. The last column shows the predictions at all longitudes combined, in which dark gray indicates that either type of fault can form over the span of longitudes at which we calculated predictions. This model predicts roughly equal numbers of right and left lateral faults at any location given a random distribution of fault azimuths.
The predictions using the SKP-type model (without obliquity) are shown in Fig. 5. In all regions, both left and right lateral faults are predicted to form with the slip direction depending on fault azimuth. Given a population of faults with random azimuths, this model should produce equal numbers of LL and RL faults at all latitudes and longitudes on Europa.

\section{Discussion}

\subsection{Matching the slip directions of observed faults}

To quantify the goodness of fit between the shell tectonics predictions and the observed faults, we made predictions of slip direction at the actual latitude, longitude, and azimuth of each observed fault (as reported in Rhoden et al., 2011). As shown in Figs. 3 and 4, the hemispheric differences in the observed fault population are better matched when obliquity is included in the tidal stress calculations. By testing predictions made with different spin pole directions against the individual observed faults, we find that an SPD of $220^{\circ}$ produces a pattern that is most consistent with the observed leading-trailing asymmetry. Using high friction, the slip directions of $70 \%$ of faults in the survey by Sarid et al. (2002) are correctly predicted at their current longitudes. Using the lower friction value, our fit improves to 75\%. Rhoden et al. (2011) tested the tidal walking model predictions against the same survey of faults. They found that an obliquity of $1^{\circ}$ and SPD of $270^{\circ}$ could account for the slip directions of $69 \%$ of faults - their best fit without appealing to longitude migration. The underlying assumptions concerning fault slip are similar between the shell tectonics model and the tidal walking model. However, Rhoden et al. (2011) only tested every $90^{\circ}$ of SPD whereas we tested at every $10^{\circ}$; that may account for the difference in optimal spin pole direction. The SKP-type model correctly predicts the slip directions of only $46 \%$ and $50 \%$ of the observed faults with eccentricity-only and with $1^{\circ}$ of obliquity $\left(\varphi=220^{\circ}\right)$, respectively. Because the SKP-type model does not produce a hemispheric asymmetry, the value of the spin pole direction does not significantly influence the goodness of fit to the observations.

In Fig. 6, we show the regions included in the strike-slip survey by Sarid et al. (2002), with different colors representing the percentage of fault slip directions accurately predicted with the shell tectonics and SKP-type models. The total number of faults in each region is also listed on shell tectonics model graphic. The numbers are the same for the SKP-type model. The shell tectonics model performs well in most regions but has trouble in some areas of mixed LL and RL faults. The SKP-type model predicts mixed faults in all regions, so it performs best in mixed regions and worst in regions where one slip direction is observed more than the other.

\subsection{Matching the azimuth distribution of observed faults}

Using shell tectonics, we calculate the net fault offsets that are predicted to accumulate during each orbit. The absolute magnitudes of these offsets depend on poorly-constrained model parameters, so we instead compare the relative accumulation rates along faults. We find that, within a given region, faults with certain azimuths are predicted to develop larger offsets per orbit than others. Faults at these azimuths should be easier to identify as having strike-slip displacement. The shell tectonics model thus predicts differences in observed fault azimuth distributions in different regions based on the relative accumulation rates. Some regions within the strike-slip survey do imply a preferred direction, such as the left lateral faults at $75^{\circ} \mathrm{N}$ that trend mostly east-west (Fig. 1). However, fault at most azimuths are observed in the equatorial regions at longitude $210^{\circ}$. 

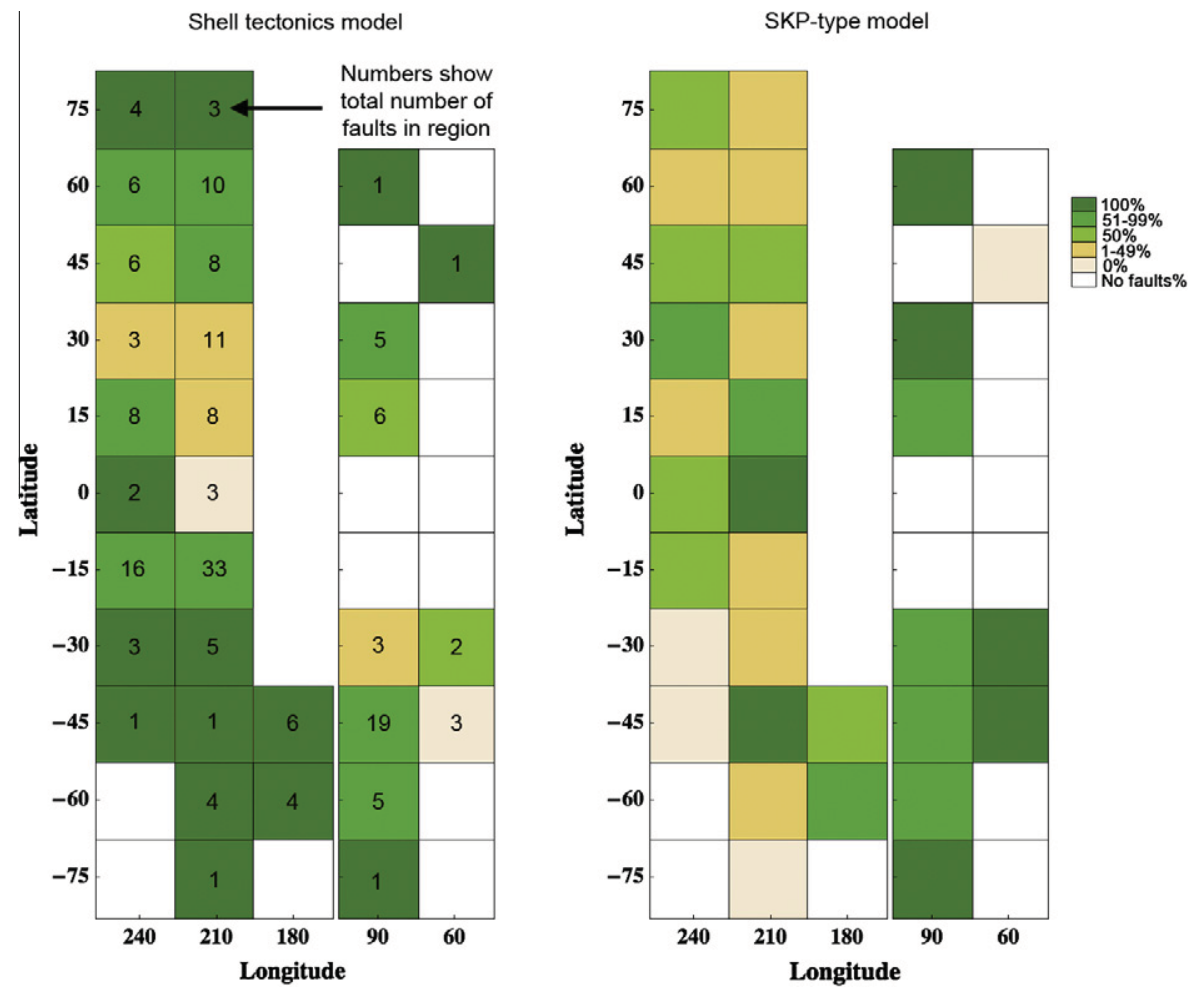

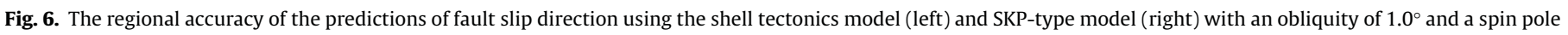
direction of $220^{\circ}$. The shading represents the percentage of faults with slip directions that were accurately predicted with each model. The number of faults in each region is shown in the shell tectonics model graphic; the numbers are the same for the SKP-type model. Regions that are not shaded contain no observed faults.

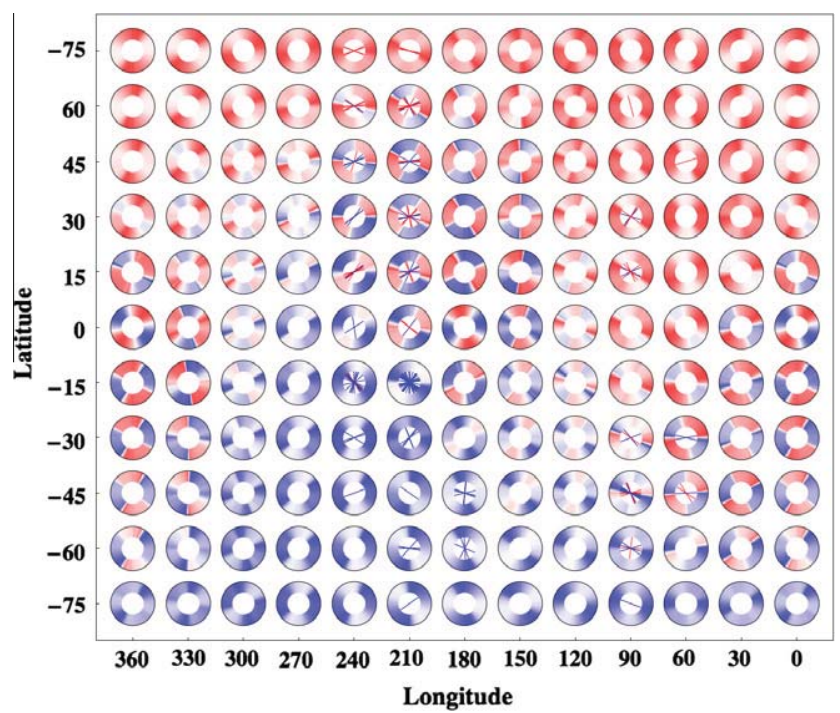

Fig. 7. Relative rates of offset accumulation for a given region using the shell tectonics model (obliquity $1^{\circ}, \operatorname{SPD} 220^{\circ}$ ) are shown in an annulus surrounding the observations from Fig. 1. The gradient of rates goes from fast accumulation of left lateral slip (bright red) to fast accumulation of right lateral slip (bright blue). White can represent slip in either direction but at a reduced pace compared to other azimuths. The differences in accumulation rate may give rise to an observation bias within the fault population. Faults that accumulate slip faster than neighboring faults should be easier to identify. (For interpretation of the references to color in this figure legend, the reader is referred to the web version of this article.)

We apply a statistical test to determine the extent to which the shell tectonics model can account for the azimuth distribution of the observed strike-slip fault population. In Fig. 7, we show the observed fault azimuths from Fig. 1 embedded within an outer annulus that displays the relative offset accumulation rate in the region from the shell tectonics model. Red represents the fastest (or largest) accumulation of LL slip, blue shows the fastest (or largest) accumulation of RL slip, and white regions may produce either LL or RL faults (as shown in Fig. 4) but at a reduced rate compared to other azimuths. These rates were calculated using the parameter values that provide the best fit to the global pattern $\left(\varepsilon=1^{\circ}\right.$, $\varphi=220^{\circ}, f=0.2$ ).

Fig. 7 shows that in some regions there should be preferred orientations at which offsets grow more rapidly. We can calculate the probability of producing the observed distribution of fault azimuths with the model shown in Fig. 7, as follows. For a given region (one circle in Fig. 7), we can construct a curve of the offset accumulation rate with azimuth. Normalizing the curve converts the rates to probabilities. Fig. 8 shows the probability curve for latitude $75^{\circ} \mathrm{N}$ and longitude $210^{\circ} \mathrm{W}$ as an example. In this region, faults of all azimuths are predicted to accumulate LL offsets; the peak in probability from $\sim 80^{\circ}$ to $\sim 135^{\circ}$ yields a prediction that more faults will be observed at these azimuths. Following this approach, it is straightforward to calculate the probability of forming each fault in the observed strike-slip population based on its binned latitude and longitude and its azimuth, rounded to the nearest degree. To compute a probability for all the faults in the survey, the probabilities for each fault are multiplied. The SKP-type model also yields net offsets along faults, so we use the same approach to test the probability of that model producing the observed fault population.

Neither the shell tectonics model nor the SKP-type model correctly predicts the slip directions of all of the observed faults. A fault with a slip direction that is not accurately predicted has zero probability of forming in the model. The probability of the model, given the observations, is proportional to the product of the individual fault probabilities, so we must either exclude the faults with 


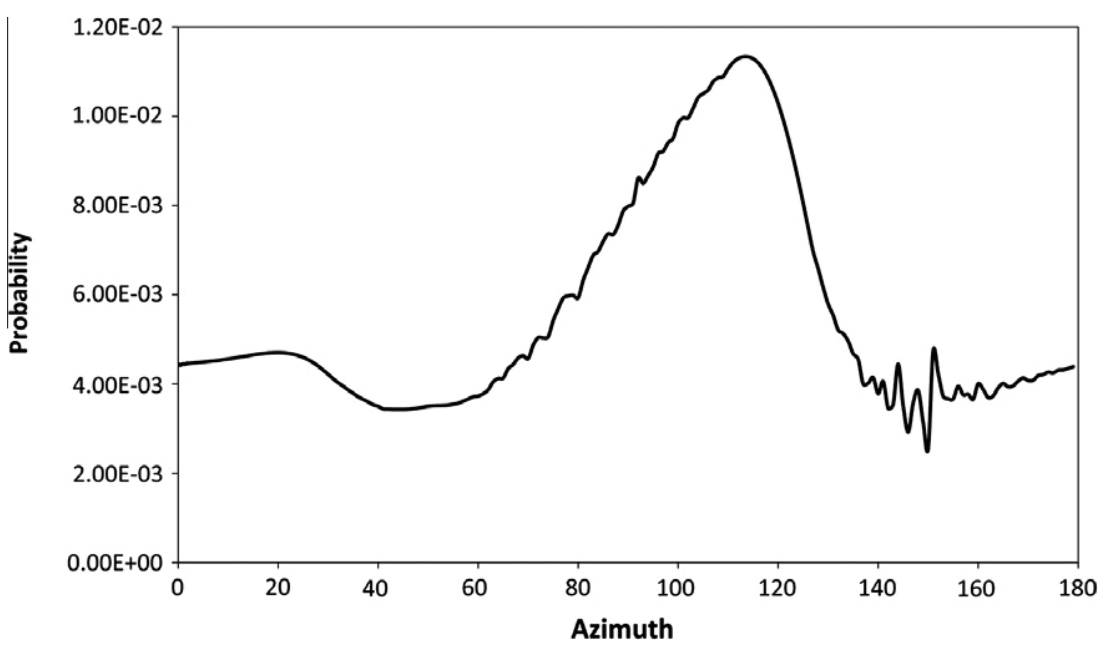

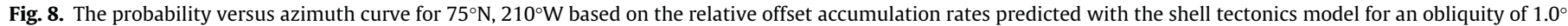
and a spin pole direction of $220^{\circ}$.

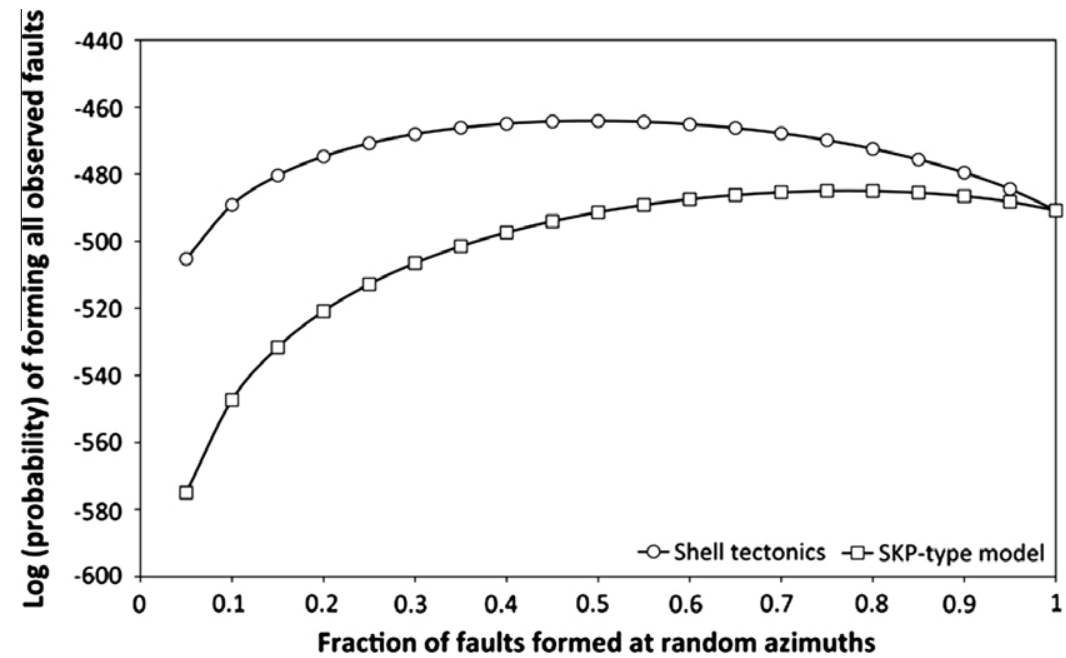

Fig. 9. The probabilities of forming the observed fault population using a two-component model for the shell tectonics (circles) and SKP (squares) models. In the twocomponent test, we assume that the slip directions and azimuths at which some offsets form are determined by the shell tectonics or SKP-type model while others are formed with random slip directions and azimuths. In the highest probability model, half the observed faults are formed via shell tectonics and half are formed randomly. The highest probability model that uses the SKP-type model requires that $75 \%$ of the observed faults formed at random orientations.

slip directions that are not accurately predicted or include a population of faults that formed with random orientations. For a fault had an equal likelihood of forming at any azimuth and either slip direction, the probability of generating that fault would be (1/ 360). Generating a specific set of randomly-selected faults would have a probability of $(1 / 360)^{n}$, where $\mathrm{n}$ is the total number of faults.

We first test the probability of forming the observed population with a two-component model, in which some faults formed via the shell tectonics or SKP-type model and some formed at random orientations through some other process. The probability of forming each observed fault, $P_{\mathrm{obs}}$, is:

$P_{\text {obs }}=f_{r} / 360+\left(1-f_{r}\right) * P_{\text {model }}$

where $f_{\mathrm{r}}$ is the fraction of faults that formed at random orientations and $P_{\text {model }}$ is the probability of forming the fault with the model (either shell tectonics or SKP-type) using the offset accumulation rates described above. As shown in Fig. 9, the two-component model with the highest probability of forming the observed fault population is one in which half the faults were created via shell tectonics and half were created at random azimuths. The SKP-type model performs best when $75 \%$ of the faults are part of the random set; the probability of this model creating the observed fault population is lower than the best overall model by a factor of $10^{20}$.

With both models, as the fraction of randomly-oriented faults approaches zero, the probability of either model forming the observed population decreases because the faults with the wrong predicted slip direction can no longer be accounted for. The population of randomly-oriented faults may reflect a population of faults that have migrated in longitude since their formation. In addition, a region may contain faults with different ages. An older fault could have built up enough offset over time to appear more prominent than a young fault even if the young fault could develop a net offset more quickly. A history of offset development and longitude migration could be pieced together with enough sequence information. The fault history would then reflect the rate of nonsynchronous rotation of the surface and the rate of growth and destruction of strike-slip offsets. Unfortunately, limited imagery makes sequencing a difficult task. Many areas simply do not have enough unambiguous cross-cutting relationships to fully constrain the formation sequence (e.g. Sarid et al., 2006). More complete sequencing should be possible if additional imagery could be 


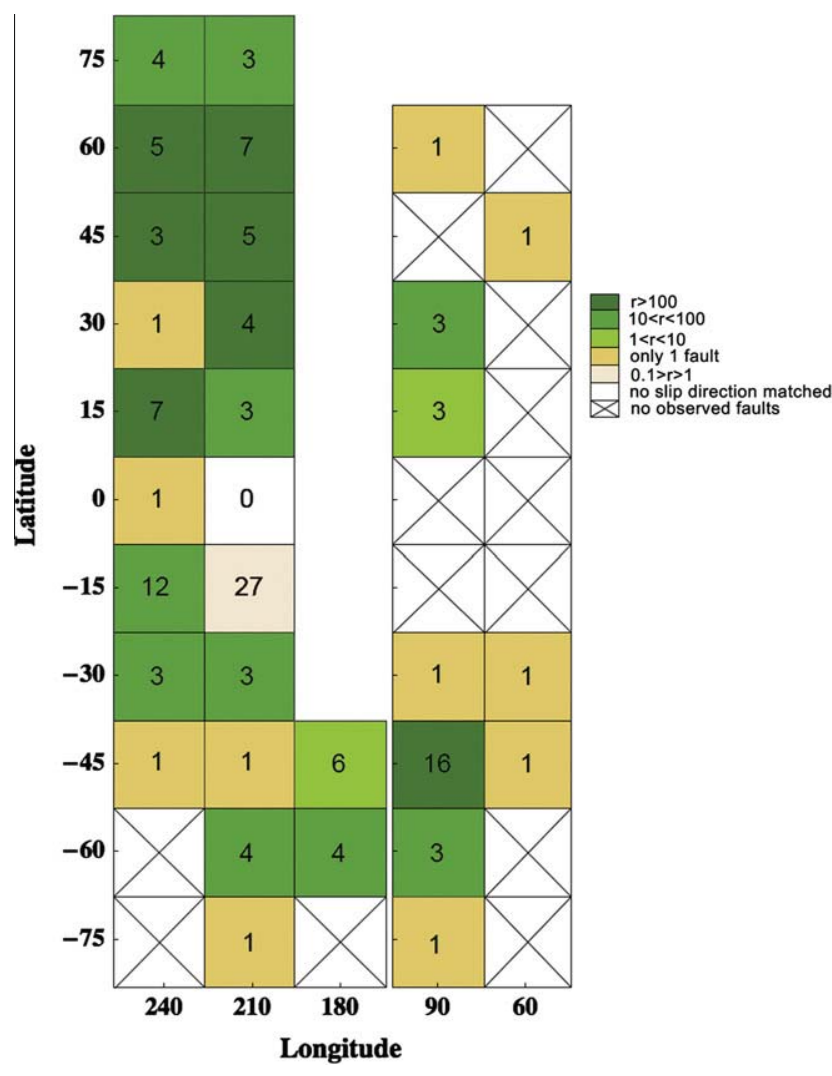

Fig. 10. The regional ratios of the probability of forming the observed faults using the shell tectonics model to that of a model that assumes random orientations and slip directions. This analysis includes only those faults with slip directions that were accurately predicted with the shell tectonics model; the number displayed within each region is the number of correctly predicted faults. Regions in shades of green represent regions in which the shell tectonics model outperforms the random model. Regions in yellow contain only one fault, so their usefulness is limited. The area shown in red is the one region that has more than one fault and is better explained by the random model than the shell tectonics model. (For interpretation of the references to color in this figure legend, the reader is referred to the web version of this article.)

obtained with at least the $\sim 250 \mathrm{~m} /$ pix resolution of the regional mapping imagery.

The two-component analysis quantifies the ability of the model to predict both the correct slip directions along faults and to generate the azimuth distribution of the faults. However, we would like to specifically investigate the potential of shell tectonics to produce the observed azimuth distribution. To do this, we include only those faults with slip directions that are accurately predicted at their current locations. We compute the probabilities of forming the observed azimuth distributions in each of the latitude/longitude bins from Fig. 1, using the offset accumulation rates predicted by shell tectonics. To compute a reference probability, we again use a model that assumes exclusively randomly-oriented faults. As described in Section 5.1, the regional probability is the product of the probabilities for each observed fault based on the offset accumulation curves. The random model probability is $(1 / 360)^{n}$, but now $\mathrm{n}$ is different for each region. This approach allows us to identify regions in which the shell tectonics model performs especially well or especially poorly.

In Fig. 10, we show the regions in which faults were mapped by Sarid et al. (2002) with a color bar that indicates the ratio of the shell tectonics probability to the random model probability. A value greater than 1 means that the observed azimuth distribution can be better reproduced by the shell tectonics model than by a random model. In this figure, the number of correctly predicted faults is listed in each region. There is only one region in which (a)

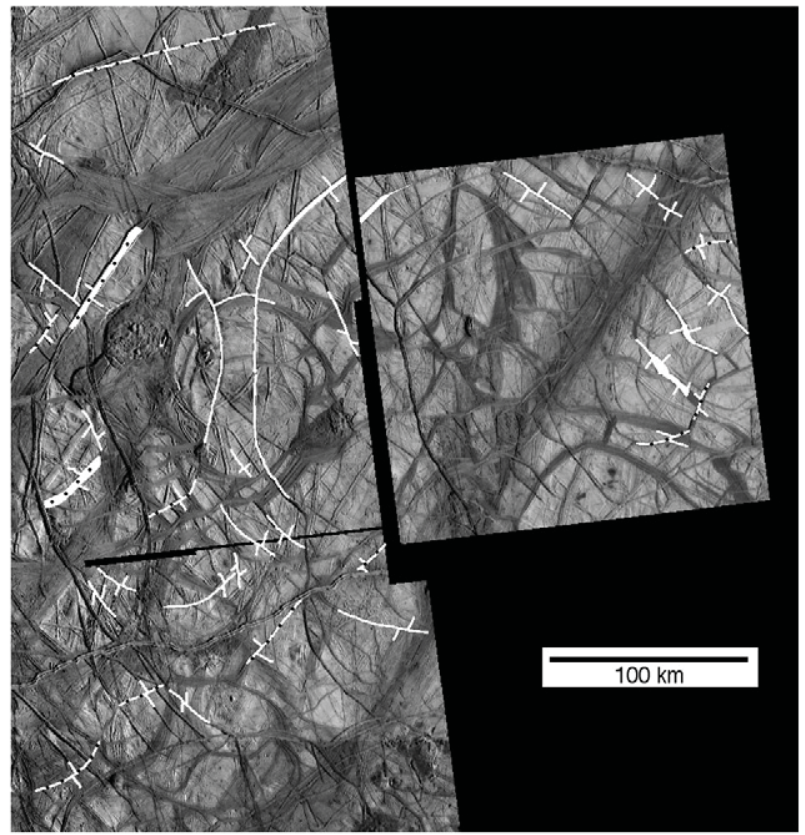

(b)

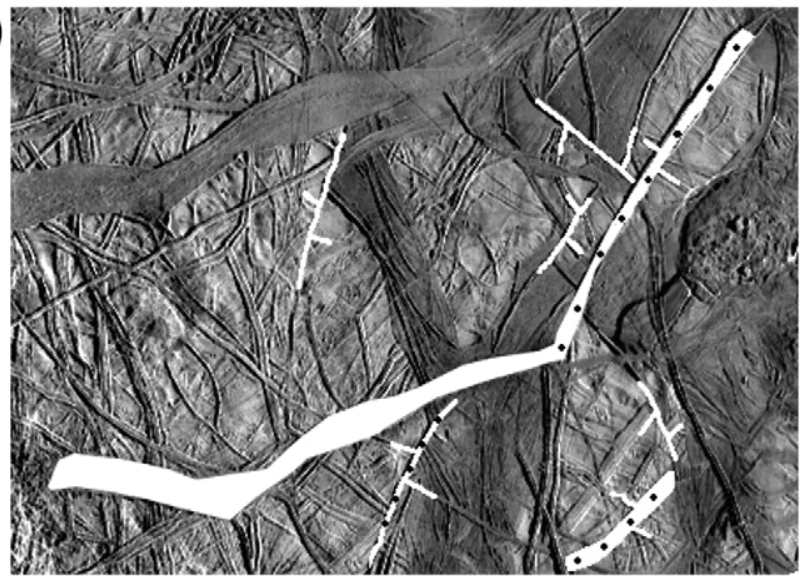

Fig. 11. (a) A complex tectonic region in which the azimuth distribution is better explained by a model that assumes random orientations than by the relative offset accumulation rates predicted by the shell tectonics model. The shell tectonics model predicts that faults with azimuths of $30-80^{\circ}$ would form offsets at a reduced rate compared with faults at other azimuths, perhaps leading to these azimuths being under-represented in the observations. It is possible that these faults formed in a different location or from a process other than tidal shear stress. Faults with slip directions and azimuths that are well-explained by the shell tectonics model are shown in white; faults with black dots have azimuths that are much less likely to be observed according to the model. (b) A fault with an azimuth that is inconsistent with the shell tectonics model at its present location. The fault is connected to a dilational band and likely formed as a result of extension along the east-west trending portion (to the left in this image, as marked by the black arrows), rather than tidal shear stress.

there is more than one fault and the observations support the random model over the shell tectonics model: longitude $210^{\circ} \mathrm{W}$, latitude $15^{\circ} \mathrm{S}$. Based on the relative offset accumulation rates in this region, we would not expect to observe offsets along faults with azimuths between $20^{\circ}$ and $80^{\circ}$. However, 12 out of the 27 faults included in the analysis of this region have azimuths in this range. The region is shown in Fig. 11a. Faults with slip directions and azimuths that are well-explained by the shell tectonics model are marked in white; faults with black dots have azimuths that are much less likely to be observed based on the model (mapping based on Sarid et al., 2002).

This region is complex; geologic mapping and reconstructions indicate that both extension and compression have contributed 
to the formation of bands in this area (Sarid et al., 2002; Patterson et al., 2006). It is possible, therefore, that some faults in this region were not formed as a direct result of tidal shear stress. For example, in Fig. 11b, we show one of the faults in more detail. This fault connects with a dilational band shown in white. As the band opened along the east-west portion (as indicated by the arrows in Fig. 11b), it would have led to right-lateral slip along the north-south portion. It appears that this fault was created as a by-product of extension, and our model correctly indicated that it was unlikely to have been produced by tidal shear stress. More in depth examination of this region may reveal that many of these faults were similarly formed. This would enhance our understanding of the complex tectonic evolution of this region and allow more accurate testing of tidally-driven strike-slip fault models.

\subsection{Additional implications}

Using the shell tectonics model and either value of the friction coefficient, we are able to generate predictions that fit the observed pattern of strike-slip faults on Europa. With obliquity $\left(\varepsilon=1^{\circ}\right)$, the differences in the leading and trailing populations can also be explained. Thus, consistent with the findings of Rhoden et al. (2011), polar wander is not necessary to explain strike-slip fault observations when the effects of obliquity are included in calculations of the stress field. Also consistent with Rhoden et al. (2011), we find that the formation timescale of strike-slip must be fast relative to the NSR timescale in order for the differences in the leading and trailing hemispheres to be preserved. As shown in the "Sum" column of Fig. 4, rapid NSR should smear out the longitudinal variations in slip direction leading to mixed LL and RL faults at most latitudes and all longitudes.Tidal stresses that include obliquity are typically about $100 \mathrm{kPa}$. If faults are only allowed to slip when in tension (such as in the tidal walking model), the compressive stress generated by the overlying ice would exceed this value below about $100 \mathrm{~m}$, and faults would be clamped throughout the entire orbit. In the shell tectonics model, as well as the SKP model, a fault can slip in both tension and compression as long as the accumulated shear stress is larger than the Coulomb failure threshold. Using our best-fit shell tectonics model $\left(\varepsilon=1^{\circ}, \varphi=220^{\circ}, f=0.2\right.$ ), faults could still slip with a compressive overburden stress corresponding to a depth of 400-500 m depending on location. Hence, based on the assumptions in the shell tectonics model, we can only really assess the behavior of this uppermost portion of the ice shell. To expand our analysis to the whole ice shell, we would need to integrate the effects of overburden stress and depth-dependent rheology into calculations of the timing and amount of slip with depth.

The statistical analyses presented in Sections 5.1 and 5.2 indicate that the shell tectonics model can better reproduce the slip directions and azimuth distributions of the observed fault population on Europa than the SKP-type model. However, it is important to point out that we have used a simplified parameterization of the model presented by Smith-Konter and Pappalardo (2008). Our work tested the underlying assumptions of how fault slip is generated, which are different between the two models. Due to these simplifications, we cannot fully assess the potential of the model presented by Smith-Konter and Pappalardo (2008) to produce offsets on Enceladus or Europa. We can conclude that the fault slip behavior they assumed is less likely to generate the observed fault population on Europa than the fault slip assumptions in the shell tectonics model.

\section{Conclusions}

Shell tectonics provides a mechanical framework to evaluate the response of faults within an elastic shell to periodic tidal stress. Shell tectonics explicitly accounts for the release of accumulated stress due to slip on the fault when computing subsequent fault stress and slip. Stress relaxation in the shell is also included explicitly. Shell tectonics includes a more physical treatment of fault mechanics than the tidal walking model (Hoppa et al., 1999) and can operate much deeper into the shell because it uses a Coulomb failure criterion to determine when and if failure occurs. However, both the shell tectonics and tidal walking models assume that, in general, slip on a fault will increase as shear stress increases and will decrease as the shear stress decreases. The model of SmithKonter and Pappalardo (2008) relies on a different assumption when evaluating fault slip. To test this fundamental difference in assumptions, we developed a simplified version of the SKP model and tested both the shell tectonics and SKP-type models against the population of observed faults on Europa from Sarid et al. (2002).

When applied to Europa, both the tidal walking (Rhoden et al., 2011) and shell tectonics models can produce global predictions of slip direction that display the same pattern as observed strike-slip faults. Consistent with previous work (Rhoden et al., 2011), we find that an obliquity of order $1^{\circ}$ can alter tidal stress such as to reproduce the differences in strike-slip populations observed in the leading and trailing hemispheres. The shell tectonics model accurately predicts the slip directions on $75 \%$ of the observed faults from the survey by Sarid et al. (2002). For comparison, the SKPtype model can fit the slip directions of $50 \%$ of observed faults.

Using the predictions of relative accumulation rates from the two models allows us to determine whether the azimuth distribution of the observed faults can be explained in addition to their slip directions. This analysis shows that both models require a population of faults that formed at random orientations ( $50 \%$ for the shell tectonics model and 75\% for the SKP-type model) in order to explain the slip directions and azimuth distribution of the observed faults. The probability of forming the observed fault population is always higher with the shell tectonics model than the SKP-type model.

We also determine the regional probabilities of forming the observed fault population in accordance with the relative accumulation rates as predicted by shell tectonics and a model in which all faults form at random azimuths. Comparison with the azimuths of observed faults strongly suggests that the differences in relative accumulation rates have led to preferred orientations within the strike-slip survey because faults with larger offsets are easier to identify. Of the 32 regions containing strike-slip faults, the shell tectonics model outperforms the random model in all regions containing more than one fault, with the exception of a complex tectonic region in which many faults may have formed through extension and compression. Further examination of this region as well as investigation of the role of overburden and other depthdependencies on fault slip would likely improve our understanding of strike-slip fault formation on Europa. Shell tectonics may also be applicable to the formation of, and motion along, the Tiger Stripe fractures on Enceladus with implications for heating and plume generation, as these processes have already been linked to tidal stress (Nimmo et al., 2007; Smith-Konter and Pappalardo, 2008; Hurford et al., 2007).

\section{Acknowledgments}

The authors would like to thank Eric Dunham for helpful discussions during the development of the shell tectonics model. This work was funded by the NASA NESSF program.

\section{Appendix A. Supplementary material}

Supplementary data associated with this article can be found, in the online version, at doi:10.1016/j.icarus.2011.12.015. 


\section{References}

Beeman, M., Durham, W.B., Kirby, S.H., 1988. Friction of ice. J. Geophys. Res. 93, 7625-7633.

Bills, B.G., Nimmo, F., Karatekin, O., Van Hoolst, T., Rambaux, N., Levrard, B., Laskar J., 2009. Rotational dynamics of Europa. In: Pappalardo, R., McKinnon, W. Khurana, K. (Eds.), Europa. Univ. Arizona Press, Tucson, pp. 119-136.

Figueredo, P.H., Greeley, R., 2000. Geologic mapping of the northern leading hemisphere of Europa from Galileo solid-state imaging data. J. Geophys. Res 105, 22629-22646.

Goldreich, P.M., Mitchell, J.L., 2010. Elastic shells of synchronously rotating moons: Implications for cracks on Europa and non-synchronous rotation on Titan. Icarus 209, 631-638.

Greenberg, R., Weidenschilling, S.J., 1984. How fast do Galilean satellites spin? Icarus 58, 186-196.

Greenberg, R. et al., 1998. Tectonic processes on Europa - Tidal stresses, mechanical response, and visible features. Icarus $135,64-78$

Hoppa, G.V., Tufts, B.R., Greenberg, R., Geissler, P., 1999. Strike-slip faults on Europa: Global shear patterns driven by tidal stress. Icarus 141, 287-298.

Hoppa, G., Greenberg, R., Tufts, B.R., Geissler, P., Phillips, C., Milazzo, M., 2000 Distribution of strike-slip faults on Europa. J. Geophys. Res. 105, 22617-22627.

Hurford, T.A., Helfenstein, P., Hoppa, G.V., Greenberg, R., Bills, B.G., 2007. Eruptions arising from tidally controlled periodic openings of rifts on Enceladus. Nature $447,292-294$

Hurford, T.A., Sarid, A.R., Greenberg, R., Bills, B.G., 2009. The influence of obliquity on Europan cycloid formation. Icarus 202, 197-215.

Kattenhorn, S.A., 2002. Nonsynchronous rotation evidence and fracture history in the Bright Plains region, Europa. Icarus 157, 490-506.

Kattenhorn, S.A., Hurford, T., 2009. Tectonics on Europa. In: Pappalardo, R. McKinnon, W., Khurana, K. (Eds.), Europa. Univ. Arizona Press, Tucson, pp. 199-236.
Melosh, H.J., 1977. Global tectonics of a Despun planet. Icarus 31, 221-243.

Melosh, H.J., 1980. Tectonic patterns on a tidally distorted planet. Icarus 43, 334 337.

Nimmo, F., Spencer, J.R., Pappalardo, R.T., Mullen, M.E., 2007. Shear heating as theorigin of the plumes and heat flux on Enceladus. Nature 447, 289-291.

Ojakangas, G.W., Stevenson, D.J., 1989. Thermal state of an ice shell on Europa. Icarus $81,220-241$.

Patterson, G.W., Head, J.W., Pappalardo, R.T., 2006. Plate motion on Europa and nonrigid behavior of the icy lithosphere: The Castalia Macula region. J. Struct. Geol. 28, 2237-2258.

Rhoden, A.R., Militzer, B., Huff, E.M., Hurford, T.A., Manga, M., Richards, M., 2010. Constraints on Europa's rotational dynamics from modeling of tidally-driven fractures. Icarus 210, 770-784.

Rhoden, A.R., Hurford, T.A., Manga, M., 2011. Strike-slip fault patterns on Europa: Obliquity or polar wander? Icarus 211, 636-647.

Riley, J., Hoppa, G.V., Greenberg, R., Tufts, B.R., Geissler, P., 2006. Distribution of chaotic terrain on Europa. J. Geophys. Res. - Planets 105, 22599-22615.

Sarid, A.R., Greenberg, R., Hoppa, G.V., Hurford, T.A., Tufts, B.R., Geissler, P., 2002. Polar wander and surface convergence of Europa's ice shell: Evidence from a survey of strike-slip displacement. Icarus 158, 24-41.

Sarid, A.R., Hurford, T., Greenberg, R., 2006. Crack azimuths on Europa - Sequencing of the northern leading hemisphere. J. Geophys. Res. - Planets 111, E08004.

Schenk, P., McKinnon, W.B., 1989. Fault offsets and lateral crustal movement on Europa: Evidence for a mobile ice shell. Icarus 79, 75-100.

Smith-Konter, B., Pappalardo, R.T., 2008. Tidally driven stress accumulation and shear failure of Enceladus' tiger stripes. Icarus 198, 435-451.

Tufts, B.R., Greenberg, R., Hoppa, G.V., Geissler, P., 1999. Astypalaea Linea: A San Andreas-sized strike-slip fault on Europa. Icarus 141, 53-64.

Wahr, J., Selvans, Z.A., Mullen, M.E., Barr, A.C., Collins, G.C., Selvans, M.M. Pappalardo, R.T., 2009. Modeling stresses on satellites due to nonsynchronous rotation and orbital eccentricity using gravitational potential theory. Icarus 200 , 188-206. 